\title{
Wearable Therapy - Detecting information from wearables and mobiles that are relevant to clinical and self-directed therapy
}

Bert Arnrich, Cem Ersoy, Oscar Mayora, Anind Dey, Nadia Berthouze, Kai Kunze

Keywords: self-directed therapy, self-quantification, pervasive health, health self-management

\section{Summary}

Introduction: This accompanying editorial provides a brief introduction into the focus theme "Wearable Therapy".

Objective: The focus theme "Wearable Therapy" aims to present contributions which target wearable and mobile technologies to support clinical and self-directed therapy.

Methods: A call for papers was announced to all participants of the "9th International Conference on Pervasive Computing Technologies for Healthcare" and was published in November 2015. A peer review process was conducted to select the papers for the focus theme.

Results: Six papers were selected to be included in this focus theme. The paper topics cover a broad range including an approach to build a health informatics research program, a comprehensive literature review of self-quantification for health self-management, methods for affective state detection of informal care givers, social-aware handling of falls, smart shoes for supporting self-directed therapy of alcohol addicts, and reference information model for pervasive health systems.

Conclusions: More empirical evidence is needed that confirms sustainable effects of employing wearable and mobile technology for clinical and self-directed therapy. Inconsistencies between different conceptual approaches need to be revealed in order to enable more systematic investigations and comparisons. 


\section{Introduction}

In the past few years, the emergence of wearables and mobiles leveraged the tracking and benchmarking of people's health and wellbeing in daily life. It is estimated that in 2016 there will be more than 3 billion mobile health app downloads worldwide [1]. The driving motivation behind is that the generated data will lead to objective knowledge about personal health indicators and the knowledge should then lead to healthy lifestyle changes. However, the number of studies which provide empirical evidence that benchmarking health indicators lead to improvements of health outcomes is still relatively low. Besides, while there is an active involvement of people who already care much about their healthy lifestyle, most of the new technologies fall short when it comes to involving the majority of people who could benefit most: the chronically ill, the old, people with sedentary lifestyle, overweight, bad dietary habits, etc. This focus theme aims to discuss challenges and directions that need to be addressed by future pervasive health systems. In 2015 the "9th International Conference on Pervasive Computing Technologies for Healthcare" was held in the historical campus of the Boğaziçi University in Istanbul, Turkey. The general chairs of the conference, the chair of the steering committee and the chairs of the technical program committee agreed to prepare a focus theme that highlights important topics that were presented during the pervasive healthcare conference. The chairs decided to prepare a focus theme that addresses contributions which target wearable and mobile technologies to support clinical and self-directed therapy.

\section{Selected Papers}

The CfP was announced to all participants of the "9th International Conference on Pervasive Computing Technologies for Healthcare" and was published in November 2015. After a peer review process, six papers were selected to be included in the focus theme "Wearable Therapy - Detecting information from wearables and mobiles that are relevant to clinical and self-directed therapy". Each of the papers is briefly presented in the paragraphs that follow.

\section{Person-generated Data in Self-quantification - A Health Informatics Research Program}

Gray et al. highlight the relatively low number of published studies which investigate and discuss empirical evidence of self-quantification's effects on health outcomes [2]. They argue that although nowadays a huge amount of internet-connected mobile, wearable and ambient systems and peer-topeer social media sites are available, most of the empirical research about the role of such health selfquantification tools is piecemeal and inconclusive. Sometimes it is even not obvious whether conventional approaches from healthcare research are even useful in studies that involve selfquantification of personal biometric data. In the paper the authors describe one approach to build a program of health informatics research. The aim of the program is to pave the way for generating rich, reliable and robust health data and their information processing in self-quantification and associated healthcare and health outcomes. New ways of integrating social media, mobile apps and wearable devices in clinical practice are offered. The research results show a variety of findings, from promising 
results and trends and significant new discoveries, to no significant effects, unforeseen effects and even potentially adverse effects. In conclusion, the authors presented their concerted efforts to build a cohesive research program within an informatics research centre. In future work, authors will extend their efforts to multicentre collaborations as a crucial next step for their centre and for the health informatics discipline as a whole.

\section{Refining the Concepts of Self-quantification Needed for Health Self-management}

Almalki et al. conducted a literature review on health self-quantification studies [3]. The aim of the review is two-fold: (1) to explore and analyse concepts that are associated with the process of incorporating self-quantification systems into users' health self-quantification activities; and (2) to explore the potentials and challenges that arise from using self-quantification systems and the generated data to maintain or promote health, and their impact on the users' certainty or confidence in taking effective actions upon these data. The authors identified 269 studies that were published between 2007 and 2015. 26 studies met the inclusion criteria: use of self-quantification technologies for health self-management; investigating users in real-world contexts or daily-life settings; providing empirical evidence; did not just propose a new solution and reported only its technical specifications. Thematic analysis was conducted for each study to find all the themes that are related to the two research aims mentioned above. The reviewed studies showed inconsistencies in the conceptualization of health self-quantification. In order to prevent misconception in health self-quantification literature and in the practical use of health self-quantification systems, the authors propose a refined concept which consists of the following four phases: personal tracking, personal analytics, personal experimentation, and personal health activation. The authors conclude, that their proposed framework reduces the inconsistencies when describing users' health self-quantification practice and that it will enable more systematic investigations to improve self-quantification systems in health selfmanagement.

\section{Detecting anxiety states when caring for people with dementia}

Miranda et al. highlight the importance of employing wearable sensing to support informal caregivers [4]. In particular, the authors present their methods to infer anxiety states of informal caregivers when caring for people with dementia. In a naturalistic enactment experiment, an elderly acted as a person suffering from dementia by showing typical behaviours such as urge to leave or disorientation which have the potential of scaring the informal caregiver. The participants had to perform a cognitive therapy session with the elderly. From the participants a number of physiological data were recorded by employing wearable sensing units such as ECG chest belt, galvanic skin response wristwatch and EEG headband. Groundtruth was obtained from participants' self-reports. In order to model the affective states of the participants, affective state diagrams were introduced which show the transitions between the affective states. By observing the transitions between the affective states, a Markov transition matrix and a Markov chain structure for internal state modelling were proposed and evaluated. The authors conclude that the presented naturalistic enactment technique allowed investigating anxiety state detection close to real live informal caregivers that care for people with dementia. 


\section{Social-aware event handling within the FallRisk project}

De Backere et al. highlight that although today there exists many methods to detect a fall, the fall handling process, which starts after a fall is identified, is usually neglected or consists only of sending a simple notification [5]. The proposed FallRisk system aims to assists in the best possible way after a fall is detected. Sensor input from wearable, mobile and ambient smart devices is considered as basis to deliver an accurate analysis of the fall incident and at the same time to support the fall handling process. Input from smart devices of the caregivers are also considered in the event handling process, e.g. context information from the caregiver like availability and location are taken into account. During a workshop the event handling process was evaluated.

\section{Unobtrusive and Continuous Monitoring of Alcohol-impaired Gait Using Smart Shoes}

Park et al. present an unobtrusive system that supports self-directed therapy of patients who require complete alcohol abstinence [6]. The proposed system employs pressure sensors that are hidden in the insoles of shoes in order to provide in-depth understanding of the spatio-temporal properties of human gait affected by alcohol. From the measured pressure values a set of gait features is extracted to discriminate between alcohol-impaired gait and normal walking. The authors argue that their proposed system avoids the inconvenience and discomfort of the widely used Breathalyzer which requires repeated measurements of the blood alcohol content at regular intervals. In comparison to self-reports, gait data is considered more accurate and less prone to fraud for the identification of intoxication. Similar to related work on gait analysis, authors report about significant inter-individual variability in the measured gait features from their participants. For example, $65 \%$ of the participants showed a decrease in gait speed with alcohol intoxication, whereas the remaining $35 \%$ increased their gait speed. In order to approach the individual differences, person-dependent classifiers are investigated and the classification accuracies are compared with general models.

\section{The MADE Reference Information Model for Interoperable Pervasive Telemedicine Systems}

Fung et al. present the development of a reference information model to support semantic interoperability of pervasive telemedicine systems [7]. The model is validated against clinical guidelines for diabetic patients. The authors highlight the differences between clinical information systems and pervasive healthcare systems, e.g. providing clinical support to patients in a free-living setting. The presented information model is tailored to support wearable and mobile healthcare technologies in daily life. The authors conclude that their proposed reference information model supports pervasive telemedicine systems that are interoperable and independent of particular clinical applications. 


\section{Conclusions}

There has never been a more exciting time for research that overlaps pervasive technology and health. In the past few years the emergence of wearables and mobiles has motivated more and more people to quantify health related aspects of their live. The generated data can lead to objective insights into health indicators and knowledge might lead to healthy lifestyle improvements. There is still a lack of empirical evidence showing that benchmarking health indicators has a sustainable effect on health outcomes. As mentioned in [2], sometime it is even not obvious whether conventional approaches from healthcare research are even useful in studies that involve self-quantification of personal biometric data. Study findings can be inconsistent from promising results and trends, to no significant effects, unforeseen effects and even potentially adverse effects. Comprehensive literature reviews like the one presented in [3] show that inconsistencies exists when describing self-quantification efforts. Their proposed framework could help to reduce such inconsistencies and might encourage more systematic investigations of pervasive user-driven health self-management. Besides developing technologies for supporting patients, the people helping the patient, e.g. informal caregivers, should be considered as well like it is shown in the research presented in [4]. The FallRisk project presented in [5] is a good example showing that even intensively researched topics like fall detection need further research efforts because important processes that occur after the main event were oversimplified in the related work. The smart shoes presented in [6] serve as a good example showing how to fulfil the essential need of unobtrusive or even hidden sensing systems in daily life. Finally, in [7] the eminent differences between clinical information systems and pervasive healthcare systems are highlighted and information models are adapted to fit the special needs of wearable and mobile healthcare technologies.

\section{References}

1. Statista. Number of mHealth app downloads worldwide from 2013 to 2016 as of November 2016. Available from https://www.statista.com/statistics/625034/mobile-health-appdownloads/

2. Gray K, Martin-Sanchez FJ, Lopez-Campos GH, Almalki M, Merolli, M. Person-generated Data in Self-quantification.

3. Almalki M, Gray K, Martin-Sanchez FJ. Refining the Concepts of Self-quantification Needed for Health Self-management - A Thematic Literature Review.

4. Miranda D, Favela J, Arnrich B. Detecting anxiety states when caring for people with dementia.

5. De Backere F, Van den Bergh J, Coppers S, Elprama S, Neli J, Verstichel S, Jacobs A, Coninx K, Ongenae $F$ and De Turck F. Social-aware event handling within the FallRisk project.

6. Park E, Lee SI, Nam HS, Garst JH, Huang A, Campion A, Arnell M, Ghalehsariand N, Park S, Chang $H$, Lu DC, Sarrafzadeh M. Unobtrusive and Continuous Monitoring of Alcohol-impaired Gait Using Smart Shoes

7. Fung NLS, Jones VM, Hermens HJ. The MADE Reference Information Model for Interoperable Pervasive Telemedicine Systems 